\title{
Paenibacillus Infection in a Pediatric Patient on ECMO
}

\author{
Wetstein Pace1, Holloway Adrian², Garber Nan²,3, Patel Ripal' \\ ${ }^{1}$ Biology, Stevenson University, Owings Mills, USA \\ ${ }^{2}$ Pediatrics, Pediatric Critical Care, PICU, University of Maryland Medical Center, Baltimore, USA \\ ${ }^{3}$ Department of Pediatrics, West Virginia School of Medicine, Morgantown, WV, USA \\ Email: rpatel@som.umaryland.edu
}

How to cite this paper: Pace, W., Adrian, H., Nan, G. and Ripal, P. (2022) Paenibacillus Infection in a Pediatric Patient on ECMO. Advances in Infectious Diseases, 12, 74-79. https://doi.org/10.4236/aid.2022.121006

Received: December 9, 2021

Accepted: March 1, 2022

Published: March 4, 2022

Copyright $\odot 2022$ by author(s) and Scientific Research Publishing Inc. This work is licensed under the Creative Commons Attribution International License (CC BY 4.0).

http://creativecommons.org/licenses/by/4.0/

\begin{abstract}
Paenibacillus is a spore forming gram positive rod that is usually found in the environment. We describe a case of a patient who contracted this organism having never left the hospital after birth. This neonate contracted Paenibacillus septic shock requiring support with venous-arterial extracorporeal membrane oxygenation (ECMO) while still admitted to the hospital after birth. This patient initially presented in severe septic shock due to a Streptococcus agalactiae infection requiring hemodynamic support with ECMO. Following treatment for the Streptococcus agalactiae infection, and while still on ECMO support, the blood culture became positive for Paenibacillus. Given that our patient had never left the hospital after birth, the finding of this organism in the blood is unique. The primary defense against this bacterium is usually the skin. The only invasive procedure this patient had was ECMO cannulation which is done in a sterile fashion. Most species are susceptible to vancomycin, clindamycin, fluoroquinolones, aminoglycosides, carbapenems, penicillin, and cephalosporins. This patient was treated with penicillin G for 14 days for the $S$. agalactiae infection prior to the blood culture being positive for Paenibacillus. More than a hundred species had been identified in the genus Paenibacillus, however, few found to cause human infection. This case is unique as it is the first pediatric case with a Paenibacillus infection and the first pediatric case where this organism which rarely causes human infection was found in the blood culture of a patient on ECMO.
\end{abstract}

\section{Keywords}

Paenibacillus, ECMO, Septic Shock

\section{Introduction}

Paenibacillus infections in humans are documented and discussed, although 
they are infrequent and generally predict a favorable patient outcome. Our patient's case is unique compared to other documented cases in regard to the age of our patient, the necessity for ECMO, and ultimately, death after resistance to several antibiotics and the development of multisystem organ failure. We report a case of Paenibacillus septic shock and multiple organ dysfunction syndromes in a term neonate cannulated onto VA-ECMO shortly after birth.

\section{Case Presentation}

Our patient was an hours-old, 39-week and 4 days female born via stat Cesarean Delivery (C-section) for a non-reassuring fetal heart rate found during routine follow-up with the obstetrics. Fetal heart tracing showed bradycardia, prompting a c-section under general anesthesia, during which meconium-stained fluid was noted. She was initially pale and floppy with no spontaneous respirations and without a palpable heart rate, which did not improve with stimulation, drying, or positive pressure ventilation. After receiving chest compressions for approximately 35 seconds, the heart rate increased to 130 - 140 beats per minute and saturations were $60 \%$. Spontaneous respirations and a stable heart rate were noted at 3 minutes of life. The patient was then placed on continuous positive airway pressure at $5 \mathrm{~cm} \mathrm{H}_{2} \mathrm{O}$.

The patient was brought to the special care nursery, where she continued to have significant respiratory distress, hypotension, and neurologic depression manifested by floppiness, pallor, and little spontaneous movement. The first arterial blood gas revealed a $\mathrm{pH} 6.97, \mathrm{PCO}_{2} 40, \mathrm{PO}_{2} 124$, bicarbonate of 9.7, base deficit of -22.1 . She was then referred to our NICU and required intubation by the transport team and initiation of a vasoactive.

Upon arrival to the Neonatal Intensive Care Unit, the infant appeared to be in severe septic shock. The first blood gas, $\mathrm{pH} 6.86, \mathrm{PCO}_{2} 39, \mathrm{PaO}_{2} 172, \mathrm{HCO}_{3} 9$, and lactate $14.1 \mathrm{mmol} / \mathrm{L}$, was obtained while on High frequency oscillatory ventilation (mean airway pressure 22, amplitude $40, \mathrm{~Hz} 8$ ), nitric oxide at $20 \mathrm{ppm}$, and $100 \% \mathrm{FiO}_{2}$. She was also receiving infusions of dopamine at $20 \mathrm{mcg} / \mathrm{kg} / \mathrm{min}$, epinephrine at $0.2 \mathrm{mcg} / \mathrm{kg} / \mathrm{min}$, and vasopressin at $0.2 \mathrm{milliunits} / \mathrm{kg} / \mathrm{min}$. The decision was made to cannulate onto VA-ECMO for refractory septic shock at 10 hours of life. She was cannulated in the right neck with an $8 \mathrm{Fr}$ internal jugular venous canula and a $12 \mathrm{Fr}$ arterial canula in the carotid. We were unable to obtain optimal flow, thus requiring additional venous drainage placement in the OR with a $14 \mathrm{Fr}$ right atrial canula via a subxiphoid approach.

The first blood culture was positive, for $S$. agalactiae on day of life one, as detected via Polymerase Chain Reaction (PCR), approximately 14 hours after birth. She was initially treated with cefotaxime and ampicillin, which was subsequently changed to penicillin $\mathrm{G}$ for 14 days. Daily blood cultures were obtained while on ECMO and were consistently negative by day of life 2. Clindamycin was also maintained for open-chest prophylaxis. Blood cultures became positive again at 10 days of life and ECMO day 10. Blood cultures were sequenced and identified 
as Paenibacillus. Despite the addition of vancomycin, blood cultures remained positive for the duration of her ECMO course, an additional 10 days.

Complications to the patient's ECMO course included severe coagulopathy, volume overload and renal failure requiring continuous renal replacement therapy, interventricular hemorrhage (grade I), status epilepticus, and right ventricular hypertrophy and dilation. During her initial course, there was significant improvement in her pulmonary function and hemodynamics. The improvements were evidenced by successful weaning off vasoactive medications, improvement in neurologic function as necessitated by progressive increases in sedation needs, and with the return of renal function. However, coinciding with the positive Paenibacillus cultures, new pulmonary infiltrates developed (Figure 1), along with the return of septic shock, which required significant vasoactive support, recurrent renal failure, liver failure and severe microvascular thrombosis resulting in significant multi-limb ischemia. Due to the evolving and progressive multi-system organ dysfunction (MODS) and inability to clear the Paenibacillus bacteremia, the patient was decannulated from ECMO. Death occurred approximately 12 hours later due to refractory hypoxemic and hypercarbic respiratory failure and septic shock. An autopsy was performed with the final cause of death considered to be multi-organ failure due to sepsis: The lungs showed changes consistent with organizing alveolar damage caused by sepsis, along with secondary changes related to ECMO therapy. Sections of bilateral lungs showed organizing diffuse alveolar damage with diffuse pneumocyte hyperplasia and widening of the alveolar walls with edema and fibroblasts, patchy hemorrhage, focal concentric intimal thickening of muscular arteries, casts of mucus and fibrin in airways focally organizing, and extending in areas into the airspaces alveolar proteinosis, septal edema, and abundant intra-alveolar macrophages.



Figure 1. Chest radiograph showing evidence of new pulmonary infiltrates. 


\section{Discussion}

Paenibacillus is a spore-forming gram-positive rod that is usually found in the environment. Given that our patient had never left the hospital after birth, the finding of this organism in her blood is unique. In a study in PLOS ONE [1], it was reported that this bacterium was predominately isolated from non-sterile body sites. The study was not able to conclude about the how the bacterium was found in these observed patients but speculated that it was due to either compromised immunity or long-term indwelling foreign bodies like catheters. The bacteria are noted to have many virulent properties that may be resistant to antibiotics and can be pathogens for humans. The primary defense against this bacterium is usually the skin. Considering the only invasive procedure our patient had was ECMO cannulation, which is performed under sterile conditions, it is surprising that the patient contracted a Paenibacillus infection.

Another case report in BMJ Journal [2] reported a wound infection due to Paenibacillus in a 79-year-old man who presented with elevated inflammatory markers post-percutaneous coronary intervention and bypass. Although sternal swabs and blood cultures were obtained during surgery and did not reveal a bacterial infection, sepsis developed post-operatively. Several retrosternal swabs subsequently detected bacteria belonging to Paenibacillus, as confirmed via PCR sequencing. It is important to note that since the patient was on empiric antibiotic treatment, PCR was the only way to confirm the cause for the bacteria. An antibiotic regiment consisting of vancomycin, clindamycin, and ciprofloxacin showed a successful outcome for the patient, and inflammatory markers subsequently returned to normal. In contrast, our patient did not respond favorably to a comparable antibiotic regiment. Most species of this bacterium are susceptible to vancomycin, clindamycin, fluoroquinolones, aminoglycosides, carbapenems, penicillins, and cephalosporins. It is important to note that our patient was treated with penicillin G for 14 days for the $S$. agalactiae infection prior to the positive blood culture confirming Paenibacillus.

In the Journal of Clinical Microbiology and Infection [3], a 54-year-old woman was admitted with mastitis with an underlying breast expander implant and found to have a Paenibacillus infection. After initially unsuccessful treatment with amoxicillin, confirmation of the bacteria via PCR was performed several months later. The PCR sequencing identified Paenibacillus from samples of peri-prosthetic fluid, capsular tissue, and a fragment of the removed expander. The patient responded successfully to the antibiotic-surgical approach.

In the Annals of Clinical and Laboratory Science [4], a case report documented another infection with this bacterium. It discussed an 80-year-old male hemodialysis patient with end-stage renal disease and multiple comorbidities who presented to an outpatient clinic a month after implantation of a left subclavian catheter with irritation at the site of the dialysis catheter. Prior to hospital admission, the patient was prescribed topical hydrocortisone, which did not alleviate the irritation. Subsequent presentation to the emergency department, 
about a month later, displayed pain at the site with blood work revealing elevation inflammatory markers. Vancomycin and gentamicin were initiated, and subsequent blood cultures identified Paenibacillus via PCR. The patient remained on the antibiotic regimen, recovered, and received an alternate catheter for continuation of dialysis treatment.

In the Journal of New Microbes and New Infections [5], the first case of a bone infection caused by Paenibacillus was reported. It described a 65-year-old man with a history of hypertension and arterial disease who presented with a bone infection post-surgical treatment from fractures to the tibia and fibula. Delayed healing of the bone from the first surgery and an unknown antibiotic regimen required a second surgery, where blood cultures confirmed growth of Paenibacillus. Amoxicillin was administered and the patient responded favorably to the treatment.

\section{Conclusion}

Interestingly, the above cases were all noted in adults, responded favorably to antibiotics, and the patients ultimately recovered. The other cases reported are those in premature neonates. Our case is unique, as it is the first case with a Paenibacillus infection found in the blood culture of a patient on ECMO: Our patient initially presented with $S$. agalactiae sepsis, and subsequently developed Paenibacillus sepsis, which resisted ampicillin, despite the report of its sensitivity to penicillins. Moreover, there is no explanation of where the patient contracted the bacterium, as it is usually found in the environment; the only invasive procedure performed was ECMO cannulation, which is done in a sterile fashion. Also unique to our patient is the non-favorable response to antibiotics and multi-system organ failure, which led to death, while most of reported cases predict patient recovery.

\section{Conflicts of Interest}

The authors declare no conflicts of interest regarding the publication of this paper.

\section{References}

[1] Celandroni, F., Salvetti, S., Gueye, S.A., Mazzantini, D., Lupetti, A., Senesi, S. and Ghelardi, E. (2016) Identification and Pathogenic Potential of Clinical Bacillus and Paenibacillus Isolates. PLOS ONE, 11, e0152831.

https://doi.org/10.1371/journal.pone.0152831

[2] Anikpeh, Y.F., Keller, P., Bloemberg, G., Grunenfelder, J. and Zinkernagel, A.S. (2010) Spacecraft Bacterium, Paenibacillus pasadenensis, Causing Wound Infection in Humans. Case Reports, 2010, bcr0620103058-bcr0620103058. https://doi.org/10.1136/bcr.06.2010.3058

[3] Marchese, A., Barbieri, R., Pesce, M., Franchelli, S. and De Maria, A. (2016) Breast Implant Infection due to Paenibacillus residui in a Cancer Patient. Clinical Microbiology and Infection, 22, 743-744. https://doi.org/10.1016/j.cmi.2016.05.012 
[4] Ouyang, J., Pei, Z., Lutwick, L., Dalal, S., Yang, L., Cassai, N., Sandhu, K., Hanna, B., Wieczorek, R.L., Bluth, M. and Pincus, M.R. (2008) Paenibacillus thiaminolyticus: A New Cause of Human Infection, Inducing Bacteremia in a Patient on Hemodialysis. Annals of Clinical \& Laboratory Science, 38, 393-400.

http://www.annclinlabsci.org/content/38/4/393.full.pdf + html

[5] Quénard, F., Aubry, C., Palmieri, M., Edouard, S., Parola, P. and Lagier, J.-C. (2016) First Case of Bone Infection Caused by Paenibacillus turicensis. New Microbes and New Infections, 11, 45-46. https://doi.org/10.1016/j.nmni.2016.02.004 